\title{
The pachytene checkpoint and its relationship to evolutionary patterns of polyploidization and hybrid sterility
}

\author{
XC Li ${ }^{1,3}, \mathrm{BC}$ Barringer ${ }^{2,3,4}$ and DA Barbash ${ }^{1}$ \\ ${ }^{1}$ Department of Molecular Biology and Genetics, Cornell University, Ithaca, NY, USA and ${ }^{2}$ Department of Ecology and Evolutionary \\ Biology, Cornell University, Ithaca, NY, USA
}

\begin{abstract}
Sterility is a commonly observed phenotype in interspecific hybrids. Sterility may result from chromosomal or genic incompatibilities, and much progress has been made toward understanding the genetic basis of hybrid sterility in various taxa. The underlying mechanisms causing hybrid sterility, however, are less well known. The pachytene checkpoint is a meiotic surveillance system that many organisms use to detect aberrant meiotic products, in order to prevent the production of defective gametes. We suggest that activation of the pachytene checkpoint may be an important mechanism contributing to two types of hybrid sterility. First, the pachytene checkpoint may form the mechanistic basis of some gene-based hybrid sterility phenotypes. Second, the pachytene checkpoint may be an important mechanism that mediates chromosomalbased hybrid sterility phenotypes involving gametes with nonhaploid (either non-reduced or aneuploid) chromosome sets. Studies in several species suggest that the strength of the
\end{abstract}

pachytene checkpoint is sexually dimorphic, observations that warrant future investigation into whether such variation may contribute to differences in patterns of sterility between male and female interspecific hybrids. In addition, plants seem to lack the pachytene checkpoint, which correlates with increased production of unreduced gametes and a higher incidence of polyploid species in plants versus animals. Although the pachytene checkpoint occurs in many animals and in fungi, at least some of the genes that execute the pachytene checkpoint are different among organisms. This finding suggests that the penetrance of the pachytene checkpoint, and even its presence or absence can evolve rapidly. The surprising degree of evolutionary flexibility in this meiotic surveillance system may contribute to the observed variation in patterns of hybrid sterility and in rates of polyploidization.

Heredity (2009) 102, 24-30; doi:10.1038/hdy.2008.84; published online 3 September 2008

Keywords: pachytene checkpoint; hybrid sterility; polyploidy; meiosis

\section{Introduction}

The genetic basis of postzygotic reproductive isolation has become clearer over the last decade. Depending on the specific mechanism involved, postzygotic isolation can be due to either genic or chromosomal incompatibility. In most animals, incompatibilities mediated by deleterious interactions among genes are thought to be the primary cause of hybrid inviability and sterility, as described by Dobzhansky $(1936,1937)$ and Muller (1940, 1942); see also Orr and Turelli (2001). In contrast, postzygotic isolation in plants often involves karyotypic changes caused by chromosomal rearrangement or genome duplication, that is, polyploidy (Werth and Windham, 1991; Rieseberg et al., 1995; Ramsey and Schemske, 1998; Comai, 2000; Lynch and Conery, 2000; Lynch and Force, 2000). However, little is known about the mechanistic basis for these incompatibilities. Here,

Correspondence: Dr DA Barbash, MBEG, Department of Molecular Biology and Genetics, Cornell University, 401 Biotech Bldg., Ithaca, NY 14853, USA.

E-mail: dab87@cornell.edu

${ }^{3}$ These authors contributed equally to this study.

${ }^{4}$ Current address: Department of Biology, University of Virginia, Charlottesville, VA 22904, USA.

Received 2 February 2008; revised 10 July 2008; accepted 12 July 2008; published online 3 September 2008 we discuss how a recent discovery from studies of meiotic regulation, the pachytene checkpoint, may contribute to our understanding of both chromosomal and gene-based hybrid sterility, and how the presence or absence of the pachytene checkpoint may be a critical factor in determining levels of polyploidization, a common route to speciation in plants. We also consider how sex-specific variation in the pachytene checkpoint may contribute to sex-specific patterns of variation in the penetrance of hybrid sterility.

\section{The pachytene checkpoint}

It is critical that the chromosomes carried by sperm, eggs and spores contain accurate representations of the parental genome. During meiosis, the maternal and paternal copies of each chromosome align with each other and form a synaptonemal complex, exchange genetic material through the process of recombination, and separate to daughter cells in the first of two meiotic cell divisions. These intricate chromosomal events are subject to errors; hence most organisms have evolved meiotic 'checkpoints' that monitor the fidelity of chromosome synapsis and the repair of DNA damage. These checkpoints cause defective meiocytes to self-destruct, thus preventing the generation of defective gametes. 
Pachytene is the stage of meiotic prophase I where chromosomes are completely aligned with their homologous partner, a process known as synapsis. In many species, including Saccharomyces cerevisiae, Drosophila melanogaster, Caenorhabditis elegans and mice, defects in recombination and/or synapsis lead to arrest during the pachytene stage, and arrested cells will eventually be eliminated (Bishop et al., 1992; Sym et al., 1993; Edelmann et al., 1996; McKee and Kleckner, 1997; Chua and Roeder, 1998; Pittman et al., 1998; Ghabrial and Schupbach, 1999; Gartner et al., 2000). This surveillance process is referred to as the pachytene checkpoint. For organisms with a strict pachytene checkpoint, triploid or trisomic individuals cannot generate gametes, and meiotic cells with pairing or recombination defects are aborted.

\section{Evidence for different mechanisms of the pachytene checkpoint in different species}

Although the pachytene checkpoint is observed in many organisms ranging from yeast to mammals, it seems that different mechanisms may be involved in different species. It has been suggested that there are at least two surveillance mechanisms that monitor meiotic chromosome metabolism in budding yeast, worms and mice. One pathway monitors recombination intermediates and the other monitors incomplete synapsis. Pachytene checkpoint genes have been most intensively studied in the budding yeast $S$. cerevisiae (reviewed in Lee and Amon, 2001). Numerous mutations have been identified that block chromosome synapsis and/or recombination and induce pachytene arrest (Roeder, 1997; Roeder and Bailis, 2000). The characterization of secondary mutations that bypass pachytene arrest in the presence of defects in recombination and synapsis has led to the identification of pachytene checkpoint genes (reviewed in Roeder and Bailis, 2000). For example, in $S$. cerevisiae, $\mathrm{PCH} 2$ was identified in a genetic screen for mutations that bypass the pachytene arrest triggered by deletions of genes such as ZIP1 (which encodes a synaptonemal complex central element component) (San-Segundo and Roeder, 1999). In this species, several genes involved in the mitotic DNA damage checkpoint also participate in the pachytene checkpoint to monitor the presence of recombination intermediates (Lydall et al., 1996; Leu and Roeder, 1999; San-Segundo and Roeder, 1999; Hochwagen et al., 2005; Hochwagen and Amon, 2006). However, in mammals, the somatic checkpoint and pachytene checkpoint might use different genes. For example, Atm-deficient somatic cells are highly resistant to radiation-induced apoptosis ( $\mathrm{Xu}$ and Baltimore, 1996), whereas meiocytes in Atm-null mice can still trigger the pachytene checkpoint and undergo apoptosis (Barlow et al., 1998).

Genetic studies of $S$. cerevisiae and the nematode C. elegans have indicated that $\mathrm{PCH} 2$ is required for a checkpoint that monitors defects in synapsis, but not DNA damage (Bhalla and Dernburg, 2005; Wu and Burgess, 2006). PCH2 orthologs have been found in many organisms (Wu and Burgess, 2006; Li and Schimenti, 2007), suggesting that synaptic checkpoints might be conserved across taxa. However, recent studies indicate that the $\mathrm{PCH} 2$ ortholog (called Trip13) in mice seems to not play a role in pachytene checkpoint control ( $\mathrm{Li}$ and
Schimenti, 2007). Furthermore, most of the other synapsis checkpoint genes identified in yeast do not have orthologs in mammals. These findings suggest that the 'pachytene checkpoint' has evolved independently in different organisms.

\section{Evidence for differences in the strictness of the pachytene checkpoint in different sexes}

Mouse meiotic mutants exhibit significant sexual dimorphism attributable to differential sensitivity of meiotic checkpoints (Hunt and Hassold, 2002). Spermatocytes are sensitive to mutations that disrupt either recombinational double-strand break repair or synapsis. In contrast, although oocytes are efficiently eliminated in response to unrepaired DNA damage, they are less sensitive to incomplete synapsis (Yuan et al., 2000; Hunt and Hassold, 2002; Barchi et al., 2005; Di Giacomo et al., 2005).

Sexual dimorphism has also been observed in triploids. Interestingly, triploid chickens (which have $\mathrm{ZW}$ chromosomes) have reversed sexual dimorphism compared with mice (which have $X Y$ chromosomes). In triploid chickens, most oocyte loss occurs in young females a few weeks after hatching, probably as a consequence of the pachytene checkpoint. However, spermatocytes from male triploids can complete meiosis without delay. Although these spermatocytes do not form fully functional sperm, they do reach the advanced stage of round spermatids (Lin et al., 1986).

The frog, Buergeria buergeri (Anura, Rhacophoridae), also has a ZW sex chromosome system and seems to have a less strict meiotic checkpoint in males. Ohta et al. (1999) found that all ZWW-type triploid frogs were females having small ovaries, and oocytes were scarce and small. However, there was little difference in the number of germ cells compared with normal diploid females, suggesting that a checkpoint is blocking meiosis. In contrast, all ZZZ-type triploid frogs were males, and their testes produced spermatozoa. These spermatozoa did show some minor defects, being somewhat long and thick when compared with spermatozoa produced by diploid males (Ohta et al., 1999). Nevertheless, the presence of spermatozoa indicates that meiosis was not aborted in these triploid males.

Because asynapsis normally triggers the pachytene checkpoint, the heterogametic sex with distinctive heteromorphic sex chromosomes must evolve a mechanism to suppress detection of asynapsis. The failure of such a mechanism would trigger the checkpoint only in the heterogametic sex, which may underlie the examples of sexual dimorphism described above. During meiotic prophase in male mammals, meiotic sex chromosome inactivation causes the $\mathrm{X}$ and $\mathrm{Y}$ chromosomes to be incorporated into the heterochromatinized XY body (also called the sex body) (Handel, 2004; Turner et al., 2006). It has been suggested that the failure of meiotic sex chromosome inactivation may trigger apoptosis of defective spermatocytes (Turner et al., 2005), since in most meiotic mutants and sterile hybrid mice, the formation of the XY body is disrupted (FernandezCapetillo et al., 2003; Turner et al., 2005; Homolka et al., 2007). In $Z W$ female birds and snakes, although the $W$ chromosome is not silenced, it has been shown that the 
unpaired regions are thickened and become partially heterochromatic (Forejt and Gregorova, 1977; Becak and Becak, 1981). Both the meiotic sex chromosome inactivation of the $\mathrm{XY}$ chromosomes and heterochromatinization of the remaining unpaired $\mathrm{ZW}$ regions may provide the mechanisms to suppress their intrinsic asynapsis (Jablonka and Lamb, 1988). Organisms with homomorphic sex chromosome do not face an intrinsic asynapsis problem, so they may not have a stricter pachytene checkpoint in the heterogametic sex. If so, then we would predict that sexual dimorphism in the degree of sterility in triploids will be more pronounced in species that have older, more differentiated sex chromosomes.

These examples bear similarity to Haldane's rule, which describes the observation that the heterogametic sex $(X Y$ or $Z W)$ is more likely to show hybrid lethality or sterility than the homogametic sex (XX or ZZ) (Haldane, 1922). Haldane's rule is likely to reflect several underlying causes, including the recessivity of hybrid incompatibility genes, the higher accumulation of such genes on the $X$ chromosome, and the higher rate of evolution of male versus female hybrid sterility genes (Wu and Davis, 1993; Masly and Presgraves, 2007). As the last explanation would counteract Haldane's rule in ZW species where females are preferentially sterile or lethal, more research is needed to understand the mechanistic basis of sterility in ZW females of species such as birds (Price and Bouvier, 2002) and Lepidoptera (Presgraves, 2002). Finally, we suggest that sexual dimorphism in the pachytene checkpoint is one promising direction for future research.

However, we note that even if sexual dimorphism is found in additional ZW species it cannot be the sole determinant of Haldane's rule. Haldane's rule does not hold in diploid Xenopus, with all described interspecific crosses producing sterile hybrid males and fertile hybrid females (Kobel, 1996). Therefore, if male Xenopus do have a weaker pachytene checkpoint, as the data from triploid taxa suggest, then this difference is clearly not causing the sex-specific differences in hybrid sterility. The violation of Haldane's rule may reflect the fact that most amphibian species do not have morphologically distinct sex chromosomes (Eggert, 2004). A recent study suggests that this preferential male sterility is caused by spermatogenesis being more sensitive to disruption in hybrids compared with oogenesis (Malone and Michalak, 2008). This finding is consistent with an earlier hypothesis for why males evolve hybrid sterility faster than females (Wu and Davis, 1993). It will be interesting to evaluate at which stage spermatogenesis is blocked in the Xenopus hybrid males and to determine whether they have a meiotic or post-meiotic defect.

\section{The pachytene checkpoint contributes to the speciation process by preventing hybrids from reproducing}

Although many cases of sterile hybrids have been described, the underlying causes of sterility have typically been investigated using classical genetic or cytological methods only, or remain entirely unknown. Indeed, the pachytene checkpoint has only recently been described and investigated on a molecular level. Here, we describe a few examples of hybrid sterility that may reflect the activity of the pachytene checkpoint. When doing so, it is important to distinguish between the terms 'sterile' and 'infertile.' Sterile refers only to situations in which no gametes are produced, whereas infertile refers to situations in which gametes are produced, but they are nonfunctional (or sub-functional). These phenotypes are likely triggered by different mechanisms. Sterility of hybrids may potentially result from activation of the pachytene checkpoint, whereas infertility of hybrids is more likely owing to other types of surveillance mechanisms, such as those that sense dosage effects because of aneuploidy or structural defects in gametes. We suggest two ways in which consideration of the pachytene checkpoint may be helpful in understanding hybrid sterility. The first is a mechanism that may be triggered by the action of hybrid incompatibility genes. We emphasize that there is unlikely to be a single mechanistic explanation for genic hybrid incompatibility phenotypes. The second may reflect a more central role of the pachytene checkpoint in sensing chromosomal incompatibilities.

\section{The pachytene checkpoint and genic incompatibilities}

Hybrid sterility in mammals has been well-studied in rodents (Forejt, 1996; Borodin et al., 1998, 2006), where it shows a clear pattern of pachytene checkpoint effects. Female hybrids are mostly fertile, whereas male hybrids are sterile with meiotic arrest around pachytene (Forejt and Ivanyi, 1974; Yoshiki et al., 1993). Aberration of chromosome pairing has been detected at the pachytene stages of hybrid male mice (Chandley, 1988). Apparently, asynapsis in the hybrids triggers checkpoint effects.

Nevertheless, detailed studies indicate that genic incompatibility also contributes to hybrid sterility. The ability of certain chromosome rearrangements to pass through meiosis depends on the genetic background (de Boer and de Jong, 1989; Speed, 1989). In hybrids between two races of the house shrew Suncus murinus that differ by five Robertsonian translocations, heterozygotes for each of the translocation chromosomes that distinguish the two parental races were found in both sterile and fertile males (Borodin et al., 1998), suggesting that chromosomal differences cannot be the sole cause of sterility. In another study, there was no apparent pairing defect in the male hybrids between Thrichomys apereoides subsp. apereoides and T. apereoides subsp. laurentius, but no spermatocytes beyond diplotene were detected, suggesting that the pachytene checkpoint is activated. This meiotic disruption phenotype segregates during backcrossing in males with homomorphic karyotypes, again suggesting a genic rather than chromosomal basis for sterility (Borodin et al., 2006). Thus far, five hybrid sterility (Hst) loci have been identified in the mouse genome (Forejt, 1996), but the genes have not been identified. Hst1-dependent sterility causes arrest at the pachytene stage (Forejt, 1996), which suggests that pachytene checkpoint pathways may be involved. It also might be the case that only certain regions in the genome, possibly the Hst loci, work as sensors to monitor the pairing, and are able to trigger the pachytene checkpoint. Other hybrid male sterility phenotypes seem to manifest post-meiotically (Good et al., 2008); hence, pachytene arrest is clearly not the sole cause of mouse hybrid sterility. 


\section{The pachytene checkpoint and chromosomal incompatibilities}

Chicken-pheasant hybrids are viable but sterile with a meiotic disruption. Stages beyond primary spermatocytes are not present, indicating the likely role of the pachytene checkpoint in hybrid sterility (Purohit and Basrur, 1977). Cytological studies indicate that the $\mathrm{Z}$ chromosomes of chickens and pheasants seem to be identical between the species, whereas the autosomal complements are radically different (Stenius et al., 1963), suggesting that chromosomal incompatibilities are responsible for hybrid sterility.

Two well-known examples of sterile hybrids are hinnies, from crossing male horses with female donkeys, and mules, from crossing female horses with male donkeys. Karyotypic dissimilarities exist between the horse (64 chromosomes), the donkey (62 chromosomes) and the ensuing hybrids (63 chromosomes), helping to explain why hybrids are typically sterile. The first mechanistic explanation of a male mule's sterility was provided by Wodsedalek (1916) who concluded that there was a block in meiosis. More recent cytological studies by electron microscopy of the mule testes have shown that spermatogenesis can only reach the pachytene stage, at which time degenerative changes occur (Hernández-Jáuregui and Márquez Monter, 1977). In female mules and hinnies, there is also a severe depletion of oocytes in the ovaries, with most oocytes being rapidly eliminated after birth (Taylor and Short, 1973), which is around the pachytene stage. These data strongly suggest that meiocytes in mules are eliminated by the mammalian pachytene checkpoint. Interestingly, there is also a block in spermatogenesis at the pachytene stage of meiosis in the testes of zebra-horse and zebra-donkey hybrids, where the karyotype differences between the parental species are even greater (King et al., 1965).

In reptiles and fish, hybrid triploids are often formed and the triploid females are sterile. For example, triploids induced in the hybrid sturgeon (Huso huso female crossed to Acipenser ruthenus male) are sterile because they are unable to complete meiosis (Omoto et al., 2005). Another example is the diploid unisexual lizard Aspidoscelis dixoni. A. dixoni normally reproduces parthenogenetically but it can mate with males from the bisexual species $A$. tigris punctilinealis. The resulting triploid female hybrids are sterile (Cole et al., 2007). Interestingly, triploid parthenogenetic species have evolved multiple times within this genus (Reeder et al., 2002). For example, in triploid Cnemidophorus uniparens females the pachytene checkpoint is overcome by premeiotic endoduplication (Cuellar, 1971). This override presumably occurs because after premeiotic endoduplication the $6 \mathrm{~N}$ meiocytes are able to synapse better than $3 \mathrm{~N}$ meiocytes.

\section{The absence of the pachytene checkpoint-a potential factor contributing to the high frequency of polyploidization in plants}

The pachytene checkpoint has not been found in plants. Most plant meiotic mutants complete meiosis and cytokinesis and produce abnormal microspores (Caryl et al., 2003). It is uncertain whether the pachytene checkpoint was initially present and then lost during plant evolution, although it does not occur in at least one basal species, the green algae Chlamydomonas reinhardtii. Triploid C. reinhardtii zygotes formed from a haploiddiploid mating can produce viable spores (Dutcher, 1988).

A major consequence of the lack of a pachytene checkpoint in plants is that, in contrast to the frequent sterility of hybrid animals, hybrid plants are generally able to produce gametes. However, a relatively large proportion of gametes produced by hybrid plants are either unbalanced (leading to infertility) or unreduced (that is, containing the somatic chromosome number). Indeed, the mean estimates of unreduced gamete production are as high as $27.52 \%$ in hybrid taxa compared with $0.56 \%$ in nonhybrids (Ramsey and Schemske, 1998). As Levin (2002) points out, this represents a nearly 50-fold increase in the production of unreduced gametes among hybrid relative to nonhybrid taxa, and is likely because of a high incidence of meiotic irregularities in the former relative to the latter. The production of unreduced gametes can result in decreased fertility when most conspecifics are producing normal, reduced gametes. In this situation, the reduction in fertility occurs because the fusion of unreduced (for example, $2 n$ ) and reduced (for example, $1 n$ ) gametes produce zygotes with uneven chromosome numbers (for example, 3n). However, if multiple individuals within a population are producing unreduced gametes, or if a single individual producing unreduced gametes reproduces through self-fertilization, viable zygotes containing twice the number of chromosomes as their parents (that is, polyploids) can be formed.

Polyploidy can arise through the doubling of chromosomes in somatic cells or through the union of unreduced gametes (deWet, 1980; Ramsey and Schemske, 1998; Levin, 2002). For many years, it was believed that somatic chromosome doubling was the predominant mode of polyploid formation, because the production of unreduced gametes was thought to be relatively rare. However, we now know that the production of unreduced gametes is more common than previously believed, and that their union is a common route to the evolution of polyploid lineages (Harlan and deWet, 1975; Bretagnolle and Thompson, 1995; Ramsey and Schemske, 1998). Unreduced gametes can arise because of errors at several points during meiosis, but the majority occur after pachytene checkpoint. Therefore, in terms of its influence on the evolution of polyploidy in plants, the lack of the pachytene checkpoint is probably most important when unreduced gametes arise as a result of what Lelley et al. (1987) and Bretagnolle and Thompson (1995) call 'premeiotic doubling'. For this reason, the lack of the pachytene checkpoint might provide one of several distinct mechanistic explanations for the ability of plants to initially establish polyploidy at high frequency relative to other taxonomic groups. In conclusion, the lack of a pachytene checkpoint can potentially contribute to polyploidy in two ways. First, it allows the survival of unreduced gametes from premeiotic doubling (or other events that produce unreduced gametes before or during pachytene). Second, it allows newly formed polyploids and hybrids to produce gametes, facilitating the 
maintenance and long-term persistence of polyploid populations.

The absence of a pachytene checkpoint may also promote intraspecific variation in plants compared with animals by increasing the tolerance for sequence divergence. In animals, the pachytene checkpoint effectively prevents interspecific gene flow. For plants, however, meiosis can proceed in hybrids even if relatively high sequence divergence causes asynapsis between chromosomes from the two parental strains or species. Thus, the absence of a pachytene checkpoint may help to explain high levels of variation in some plants, for example, the finding that two lineages of maize are, on average, as genetically diverged as humans and chimpanzees (Buckler et al., 2006).

\section{Do animal polyploids lack the pachytene checkpoint?}

Polyploidy is relatively rare in most groups of animals. However, there is increasing evidence that in some groups of animals polyploidy is more frequent than previously believed (Bogart, 1980; Lokki and Saura, 1980; Schultz, 1980; Otto and Whitton, 2000; Gallardo et al., 2004; Le Comber and Smith, 2004; Mable, 2004). As the pachytene checkpoint effectively eliminates the production of unreduced gametes in animals, we might expect that, relative to plants, a higher proportion of polyploid animals are formed through mitotic duplication (that is, endoduplication). Interestingly, this prediction is consistent with the observation that the majority of polyploid animals reproduce parthenogenetically, and the evolution of parthenogenesis in animals typically predates polyploidy (Suomalainen et al., 1987; Otto and Whitton, 2000). These findings raise two major questions. First, what accounts for the general difference in rates of polyploidization between plants and animals? Second, what accounts for the relatively high incidence of polyploidy in certain animal groups?

A number of explanations have been suggested to account for these patterns (Muller, 1925; Orr, 1990; Mable, 2004). We suggest that the lack of a pachytene checkpoint in plants is an additional explanation for the first question of why polyploidy is generally more common among plants. How do we account for the presence of polyploid animals among some insects, reptiles, amphibians and fish? One possibility is that these polyploid animals have lost the pachytene checkpoint genes that are present in their non-polyploid sister taxa. This scenario would imply that polyploid animals would proceed through meiosis even when subject to various meiotic defects. Indeed, as we have noted, the pachytene checkpoint is sexually dimorphic in some species, which demonstrates that variability in the penetrance of the pachytene checkpoint can be tolerated within species.

Alternatively, these animal polyploids may have evolved mechanisms to override the ability of the pachytene checkpoint to detect or respond to the presence of non-haploid gametes, as in the case of premeiotic endoduplication. This scenario would also imply that polyploids retain the ability to arrest meiotic products that have received other types of DNA damage. Analysis of the pachytene checkpoint in the model organisms zebrafish (Danio rerio) and Xenopus frogs (Xenopus laevis) will provide useful data for launching comparative studies of polyploid fish and amphibians to investigate whether and how they have evolved the ability to produce non-haploid gametes.

In conclusion, the pachytene checkpoint is not only a surveillance system in meiosis. We suggest that the presence or absence of a pachytene checkpoint has a profound influence on the evolutionary strategies of different eukaryotic groups, and of the different sexes. On the basis of evidence reviewed here, we can make two conclusions. First, the pachytene checkpoint is the underlying mechanism for hybrid sterility in some cases, but more cytological evidence on additional hybrids is required to assess the generality of this finding. Second, the absence of a pachytene checkpoint in plants might influence the production and/or survival of unreduced gametes and allow newly formed polyploids and hybrids to produce viable gametes, facilitating the maintenance and long-term persistence of polyploid populations. Further research on polyploid animals is one direction that we suggest will help to test this hypothesis. The finding that different organisms seem to use different genes to execute the pachytene checkpoint underscores the evolutionary lability of this meiotic surveillance system, and calls for the further study of the molecular mechanisms controlling the pachytene checkpoint in different organisms.

\section{Acknowledgements}

We thank the anonymous reviewers and the Associate Editor for helpful suggestions.

\section{References}

Barchi M, Mahadevaiah S, Di Giacomo M, Baudat F, de Rooij DG, Burgoyne PS et al. (2005). Surveillance of different recombination defects in mouse spermatocytes yields distinct responses despite elimination at an identical developmental stage. Mol Cell Biol 25: 7203-7215.

Barlow C, Liyanage M, Moens PB, Tarsounas M, Nagashima K, Brown K et al. (1998). Atm deficiency results in severe meiotic disruption as early as leptonema of prophase I. Development 125: 4007-4017.

Becak M, Becak W (1981). Behaviour of the ZW sex bivalent in the snake Bothrops jararaca. Chromosoma 83: 289-293.

Bhalla N, Dernburg AF (2005). A conserved checkpoint monitors meiotic chromosome synapsis in Caenorhabditis elegans. Science 310: 1683-1686.

Bishop DK, Park D, Xu L, Kleckner N (1992). DMC1: a meiosisspecific yeast homolog of $E$. coli recA required for recombination, synaptonemal complex formation, and cell cycle progression. Cell 69: 439-456.

Bogart JP (1980). Evolutionary implications of polyploidy in amphibians and reptiles. In: Lewis WH (ed). Polyploidy: Biological Relevance. Plenum Press: New York. pp 341-378.

Borodin PM, Barreiros-Gomez SC, Zhelezova AI, Bonvicino CR, D'Andrea PS (2006). Reproductive isolation due to the genetic incompatibilities between Thrichomys pachyurus and two subspecies of Thrichomys apereoides (Rodentia, Echimyidae). Genome 49: 159-167.

Borodin PM, Rogatcheva MB, Zhelezova AI, Oda S (1998). Chromosome pairing in inter-racial hybrids of the house musk shrew (Suncus murinus, Insectivora, Soricidae). Genome 41: 79-90. 
Bretagnolle F, Thompson JD (1995). Gametes with the somatic chromosome number: mechanisms of their formation and role in the evolution of polyploid plants. New Phytologist 129: $1-22$.

Buckler ES, Gaut BS, McMullen MD (2006). Molecular and functional diversity in maize. Current Opinion in Plant Biology 9: $172-176$.

Caryl AP, Jones GH, Franklin FC (2003). Dissecting plant meiosis using Arabidopsis thaliana mutants. J Exp Bot 54: 25-38.

Chandley AC (1988). Meiotic studies and infertility. In: Daniel A (ed). Cytogenetics of Autosomal Rearrangments. Alan R. Liss, Inc.: New York. pp 361-382.

Chua PR, Roeder GS (1998). Zip2, a meiosis-specific protein required for the initiation of chromosome synapsis. Cell 93: 349-359.

Cole CJ, Painter CW, Dessauer HC, Taylor HL (2007). Hybridization between the endangered unisexual graycheckered whiptail lizard (Aspidoscelis dixoni) and the bisexual Western whiptail lizard (Aspidoscelis tigris) in southwestern New Mexico. American Museum Novitates 3555: $1-31$.

Comai L (2000). Genetic and epigenetic interactions in allopolyploid plants. Plant Mol Biol 43: 387-399.

Cuellar O (1971). Reproduction and the mechanism of meiotic restitution in the parthenogenetic lizard Cnemidophorus uniparens. J Morphol 133: 139-165.

de Boer P, de Jong JH (1989). Chromosome pairing and fertility in mice. In: Gillies C (ed). Fertility and Chromosome Pairing: Recent Studies in Plants and Animals. CRC Press Inc: Boca Raton, FL. pp 37-76.

deWet JM (1980). Origins of polyploids. In: Lewis WH (ed). Polyploidy: Biological Relevance. Plenum Press: New York. pp 3-15.

Di Giacomo M, Barchi M, Baudat F, Edelmann W, Keeney S, Jasin M (2005). Distinct DNA-damage-dependent and -independent responses drive the loss of oocytes in recombination-defective mouse mutants. Proc Natl Acad Sci USA 102: 737-742.

Dobzhansky T (1936). Studies on hybrid sterility. II. Localization of sterility factors in Drosophila pseudoobscura hybrids. Genetics 21: 113-135.

Dobzhansky T (1937). Genetics and the Origin of Species. Columbia University Press: New York.

Dutcher SK (1988). Nuclear fusion-defective phenocopies in Chlamydomonas reinhardtii: mating-type functions for meiosis can act through the cytoplasm. Proc Natl Acad Sci USA 85: 3946-3950.

Edelmann W, Cohen PE, Kane M, Lau K, Morrow B, Bennett S et al. (1996). Meiotic pachytene arrest in MLH1-deficient mice. Cell 85: 1125-1134.

Eggert C (2004). Sex determination: the amphibian models. Reprod Nutr Dev 44: 539-549.

Fernandez-Capetillo O, Mahadevaiah SK, Celeste A, Romanienko PJ, Camerini-Otero RD, Bonner WM et al. (2003). $\mathrm{H} 2 \mathrm{AX}$ is required for chromatin remodeling and inactivation of sex chromosomes in male mouse meiosis. Dev Cell 4: 497-508.

Forejt J (1996). Hybrid sterility in the mouse. Trends Genet 12: 412-417.

Forejt J, Gregorova S (1977). Meiotic studies of translocations causing male sterility in the mouse. I. Autosomal reciprocal translocations. Cytogenet Cell Genet 19: 159-179.

Forejt J, Ivanyi P (1974). Genetic studies on male sterility of hybrids between laboratory and wild mice (Mus musculus L.). Genet Res 24: 189-206.

Gallardo MH, Kausel G, Jimenez A, Bacquet C, Gonzalez C, Figueroa $J$ et al. (2004). Whole genome duplications in South American desert rodents (Octodontidae). Biological Journal of the Linnean Society 82: 443-451.

Gartner A, Milstein S, Ahmed S, Hodgkin J, Hengartner MO (2000). A conserved checkpoint pathway mediates DNA damage-induced apoptosis and cell cycle arrest in C. elegans. Mol Cell 5: 435-443.

Ghabrial A, Schupbach T (1999). Activation of a meiotic checkpoint regulates translation of Gurken during Drosophila oogenesis. Nat Cell Biol 1: 354-357.

Good JM, Handel MA, Nachman MW (2008). Asymmetry and polymorphism of hybrid male sterility during the early stages of speciation in house mice. Evolution Int $J$ Org Evolution 62: 50-65.

Haldane JBS (1922). Sex ratio and unisexual sterility in hybrid animals. J Genetics 12: 101-109.

Handel MA (2004). The XY body: a specialized meiotic chromatin domain. Exp Cell Res 296: 57-63.

Harlan JR, deWet JM (1975). On O. Winge and a prayer: the origins of polyploidy. Botanical Review 41: 361-390.

Hernández-Jáuregui P, Márquez Monter H (1977). Fine structure of mule testes: light and electron microscopy study. Am J Vet Res 38: 443-447.

Hochwagen A, Amon A (2006). Checking your breaks: surveillance mechanisms of meiotic recombination. Curr Biol 16 R217-R228

Hochwagen A, Tham WH, Brar GA, Amon A (2005). The FK506 binding protein Fpr3 counteracts protein phosphatase 1 to maintain meiotic recombination checkpoint activity. Cell 122: 861-873.

Homolka D, Ivanek R, Capkova J, Jansa P, Forejt J (2007). Chromosomal rearrangement interferes with meiotic $X$ chromosome inactivation. Genome Res 17: 1431-1437.

Hunt PA, Hassold TJ (2002). Sex matters in meiosis. Science 296 : 2181-2183.

Jablonka E, Lamb MJ (1988). Meiotic pairing constraints and the activity of sex chromosomes. I Theor Biol 133: 23-36.

King JM, Short RV, Mutton DE, Hamerton JL (1965). Reproductive physiology of male zebra-horse and zebra-donkey hybrids. Journal of Reproduction and Fertility 9: 391-393.

Kobel HR (1996). Allopolyploid speciation. In: Tindley RC, Kobel HR (eds). The Biology of Xenopus. Clarendon: Oxford. pp 391-401.

Le Comber SC, Smith C (2004). Polyploidy in fishes: patterns and processes. Biological Journal of the Linnean Society 82: 431-442.

Lee B, Amon A (2001). Meiosis: how to create a specialized cell cycle. Curr Opin Cell Biol 13: 770-777.

Lelley T, Mahmoud AA, Lein V (1987). Genetics and cytology of unreduced gametes in cultivated rye (Secale cereale L.) Genome 29: 635-638.

Leu JY, Roeder GS (1999). The pachytene checkpoint in S. cerevisiae depends on Swe1-mediated phosphorylation of the cyclin-dependent kinase Cdc28. Mol Cell 4: 805-814.

Levin DA (2002). The Role of Chromosomal Change in Plant Evolution. Oxford University Press: New York.

Li XC, Schimenti JC (2007). Mouse pachytene checkpoint 2 (trip13) is required for completing meiotic recombination but not synapsis. PLoS Genet 3: e130.

Lin M, Thorne MH, Martin IC, Sheldon BL (1986). Histlogy of the gonads of triploid fowls. Paper presented at: Proceedings of the 18th Annual Conference of the Australian Society of Reproduction Biology. Brisbane: Australia.

Lokki J, Saura A (1980). Polyploidy in insect evolution. In: Lewis WH (ed). Polyploidy: Biological Relevance. Plenum Press: New York. pp 277-312.

Lydall D, Nikolsky Y, Bishop DK, Weinert T (1996). A meiotic recombination checkpoint controlled by mitotic checkpoint genes. Nature 383: 840-843.

Lynch M, Conery JS (2000). The evolutionary fate and consequences of duplicate genes. Science 290: 1151-1155.

Lynch M, Force AG (2000). The origin of interspecific genomic incompatibility via gene duplication. Am Nat 156 590-605.

Mable BK (2004). Why polyploidy is rarer in animals than in plants': myths and mechanisms. Biological Journal of the Linnean Society 82: 453-466. 
Malone JH, Michalak P (2008). Physiological sex predicts hybrid sterility regardless of genotype. Science 319: 59.

Masly JP, Presgraves DC (2007). High-resolution genome-wide dissection of the two rules of speciation in Drosophila. PLoS Biol 5: e243.

McKee AH, Kleckner N (1997). Mutations in Saccharomyces cerevisiae that block meiotic prophase chromosome metabolism and confer cell cycle arrest at pachytene identify two new meiosis-specific genes SAE1 and SAE3. Genetics 146: 817-834.

Muller HJ (1925). Why polyploidy is rarer in animals than in plants. The American Naturalist 59: 346-353.

Muller HJ (1940). Bearings of the 'Drosophila' work on systematics. In: Huxley J (ed). The New Systematics. The Clarendon Press: Oxford. pp 185-268.

Muller HJ (1942). Isolating mechanisms, evolution and temperature. Biol Symp 6: 71-125.

Ohta S, Sumida M, Nishioka M (1999). Sex-determining mechanism in Buergeria buergeri (Anura, Rhacophoridae). III. Does the ZZW triploid frog become female or male? J Exp Zool 283: 295-306.

Omoto N, Maebayashi M, Adachi S, Arai K, Yamauchi K (2005). Sex ratios of triploids and gynogenetic diploids induced in the hybrid sturgeon, the bester (Huso huso female $\mathrm{x}$ Acipenser ruthenus male). Aquaculture 245: 39-47.

Orr HA (1990). 'Why polyploidy is rarer in animals than in plants': revisited. The American Naturalist 136: 759-770.

Orr HA, Turelli M (2001). The evolution of postzygotic isolation: accumulating Dobzhansky-Muller incompatibilities. Evolution Int J Org Evolution 55: 1085-1094.

Otto SP, Whitton J (2000). Polyploid incidence and evolution. Annual Review of Genetics 34: 401-437.

Pittman DL, Cobb J, Schimenti KJ, Wilson LA, Cooper DM, Brignull E et al. (1998). Meiotic prophase arrest with failure of chromosome synapsis in mice deficient for $D m c 1$, a germlinespecific RecA homolog. Mol Cell 1: 697-705.

Presgraves DC (2002). Patterns of postzygotic isolation in Lepidoptera. Evolution Int J Org Evolution 56: 1168-1183.

Purohit VD, Basrur PK (1977). Effect of extended photoperiod on the spermatogenic activity of chicken-pheasant hybrids. Br Poult Sci 18: 651-655.

Price TD, Bouvier MM (2002). The evolution of F1 postzygotic incompatibilities in birds. Evolution Int J Org Evolution 56: 2083-2089.

Ramsey J, Schemske DW (1998). Pathways, mechanisms, and rates of polyploid formation in flowering plants. Annu Rev Ecol Syst 29: 467-501.

Reeder TW, Colle CJ, Dessauer HC (2002). Phylogenetic relationships of whiptail lizards of the genus Cnemidophorus (Squamata: Teiidae): a test of monophyly, reevaluation of karyotypic evolution, and review of hybrid origins. American Museum Novitates 3365: 1-61.

Rieseberg LH, Vanfossen C, Desrochers AM (1995). Hybrid speciation accompanied by genomic reorganization in wild sunflowers. Nature 375: 313-316.
Roeder GS (1997). Meiotic chromosomes: it takes two to tango. Genes Dev 11: 2600-2621.

Roeder GS, Bailis JM (2000). The pachytene checkpoint. Trends Genet 16: 395-403.

San-Segundo PA, Roeder GS (1999). Pch2 links chromatin silencing to meiotic checkpoint control. Cell 97: 313-324.

Schultz RJ (1980). Role of polyploidy in the evolution of fishes. In: Lewis WH (ed). Polyploidy: Biological Relevance. Plenum Press: New York. pp 313-340.

Speed R (1989). Heterologous pairing and fertility in humans. In: Gillies C (ed). Fertility and Chromosome Pairing: Recent Studies in Plants and Animals. CRC Press: Boca Raton, FL. pp 1-35.

Stenius C, Christian LC, Ohno S (1963). Comparative cytological study of Phasianus colchicus, Meleagris gallopavo, and Gallus domesticus. Chromosoma 13: 515-520.

Suomalainen E, Saura A, Lokki J (1987). Cytology and Evolution in Parthenogenesis. CRC Press Inc.: Boca Raton, Florida.

Sym M, Engebrecht JA, Roeder GS (1993). ZIP1 is a synaptonemal complex protein required for meiotic chromosome synapsis. Cell 72: $365-378$.

Taylor MJ, Short RV (1973). Development of the germ cells in the ovary of the mule and hinny. J Reprod Fertil 32: 441-445.

Turner JM, Mahadevaiah SK, Ellis PJ, Mitchell MJ, Burgoyne PS (2006). Pachytene asynapsis drives meiotic sex chromosome inactivation and leads to substantial postmeiotic repression in spermatids. Dev Cell 10: 521-529.

Turner JM, Mahadevaiah SK, Fernandez-Capetillo O, Nussenzweig A, Xu X, Deng CX et al. (2005). Silencing of unsynapsed meiotic chromosomes in the mouse. Nat Genet 37: 41-47.

Werth C, Windham MD (1991). A model for divergent, allopatric speciation of polyploid pteridophytes resulting from silencing of duplicate gene expression. Am Nat 137: 515-526.

Wodsedalek JE (1916). Causes of sterility in the mule. Biol Bull 30: $1-56$.

Wu C-I, Davis AW (1993). Evolution of postmating reproductive isolation: the composite nature of Haldane's rule and its genetic bases. Am Nat 142: 187-212.

Wu HY, Burgess SM (2006). Two distinct surveillance mechanisms monitor meiotic chromosome metabolism in budding yeast. Curr Biol 16: 2473-2479.

Xu Y, Baltimore D (1996). Dual roles of ATM in the cellular response to radiation and in cell growth control. Genes Dev 10: 2401-2410.

Yoshiki A, Moriwaki K, Sakakura T, Kusakabe M (1993). Histological studies on male sterility of hybrids between laboratory and wild mouse strains. Development, Growth $\mathcal{E}$ Differentiation 35: 271-281.

Yuan L, Liu JG, Zhao J, Brundell E, Daneholt B, Hoog C (2000). The murine SCP3 gene is required for synaptonemal complex assembly, chromosome synapsis, and male fertility. Mol Cell 5: 73-83. 